\title{
Behavioural study of COVID-19 symptom showers.
}

\author{
Pravar Jain ${ }^{1}$ \\ ${ }^{1}$ Affiliation not available
}

June 22, 2020

\begin{abstract}
This research was aimed at understanding the psychology of citizens to get tested and to follow prevention measures when they show COVID-19 symptoms. It analyses the monetary and psychological perspective. It was found that $75 \%$ of the symptomatic individuals did not follow isolation measures, and $80 \%$ of symptomatic participants chose to not test, solely based on the decreased severity of symptoms. 7 respondents showed severe symptoms but restrained themselves from taking a test due to the high cost of testing for Covid-19.
\end{abstract}

\section{Introduction:}

Covid-19 is an illness in which the main method of transmission is the contact between a virus carrier (host) and a susceptible host. A Covid-19 patient can infect 406 people in 30 days if preventive measures such as isolation and social distancing are not implemented.

Asymptomatic patients, who don't show symptoms but are carriers of the virus, can also transmit it at the same level.

\section{Aim of research:}

With this research, I am aiming to understand the psychology of a random sample of the population, in regards to Covid-19 testing. I want to infer, if a person experiences symptoms which are very common to Covid-19, would they get tested, follow isolation protocols or not, and their reasoning behind it.

\section{Method and demographic:}

65 acquaintances were chosen, in a 5:4:1 ratio of ages 0-30, 30-60, and above 60 years. They were made to answer a survey, online. All questions were close ended, multiple choice questions.

The participants who answered to have experienced Covid-19 symptoms, were further questioned if they had taken a test or not.

If not, they were asked to answer with the reason for not being tested. All respondents were from India.

\section{Below are the question and responses visualized:}

\section{Q1. What is your age?}
a) 0-30 years
b) $30-60$ years
c)60-100 years 
What is your age?

63 responses

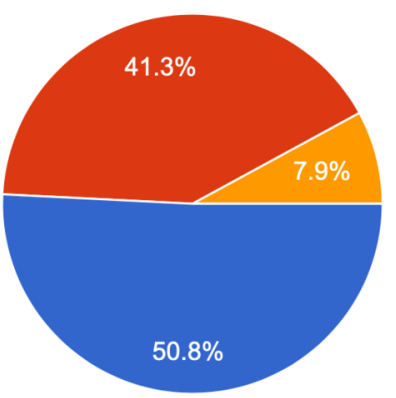

0-30 years

$30-60$ years

60-100 years

About $51 \%$ of the participants were aged $0-30$ years, $41 \%$ are $30-60$ years of age, and $8 \%$ were above 60 years in age.

\section{Q2. Have you had any of these illnesses in the past 3 months? (Select all that apply)}
a)Running cold
b)Fever
c)Dry cough
d)Loss of sense of smell or taste
e) None

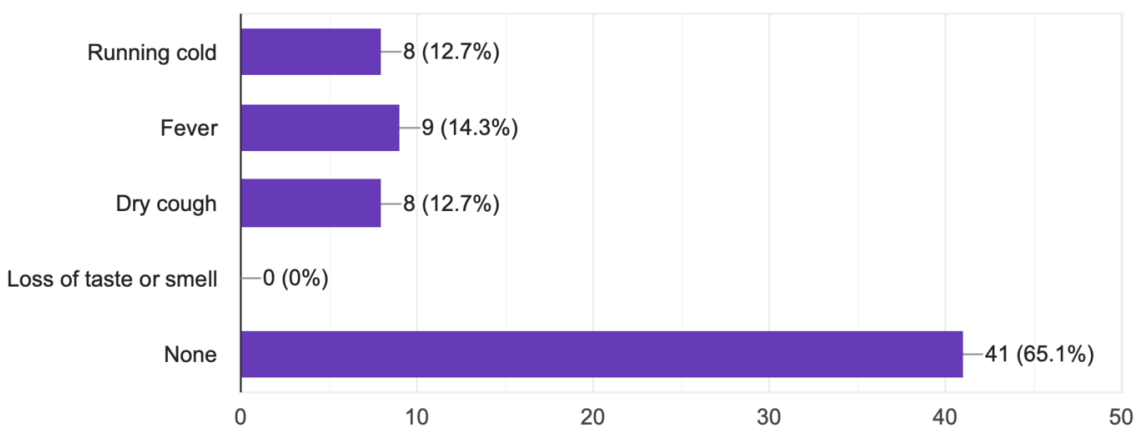

$65 \%$ of the participants reported as having no illness. A combined $35 \%$ of participants had an illness that is one of the most common symptom of Covid-19.

3. Did you self isolate yourself when you were ill (If you were)
a) Yes
b) No 
Did you self isolate yourself when you were ill?

27 responses

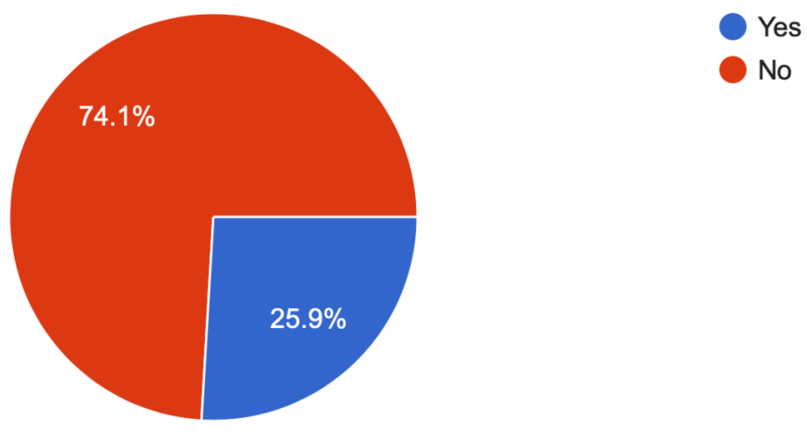

[Edited pie chart after removing response errors, where participants reporting no symptoms had answer this question]

$74 \%$ of participants who reported being ill, did not follow self-isolation measures. Only $26 \%$ of reported symptomatic participants did.

4. Have you been tested for Covid-19?

a) Yes

b) No

\section{Have you been tested for Covid-19?}

63 responses

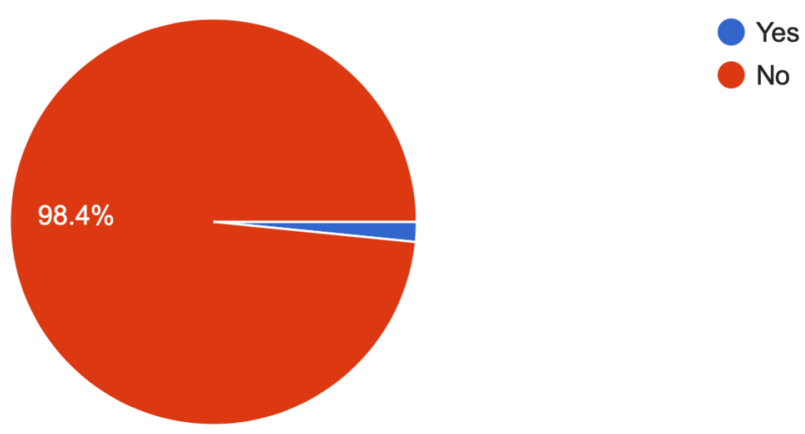

Across 63 participants, only 1 has been tested for Covid-19. This is among 27 participants who showed very common Covid-19 symptoms, yet were not tested for Covid-19. 


\section{Q5. If no, what was the reason?}

a) Symptoms weren't severe enough

b) Symptoms were severe but you were restrained by the cost of the test

c) You were denied testing

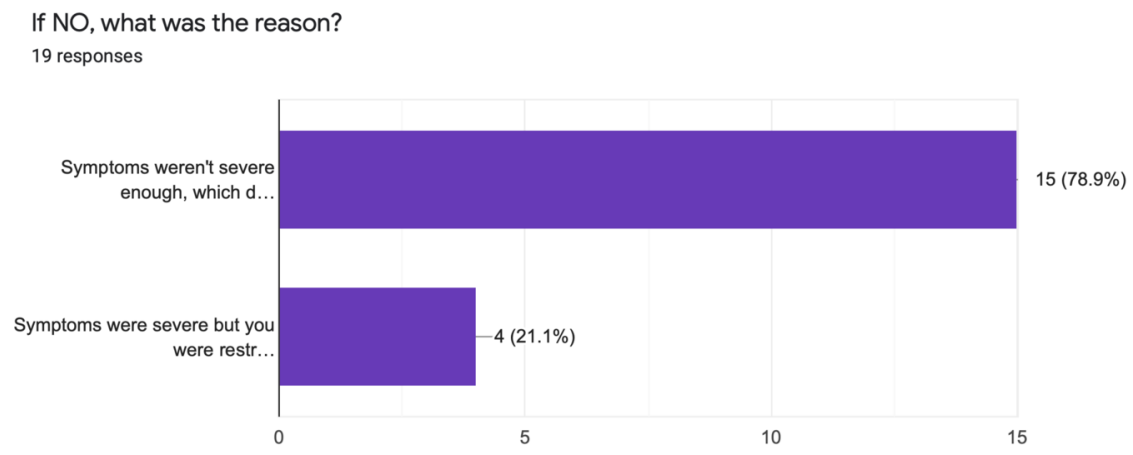

[Adjusted chart after removing response errors where non-symptomatic respondents had answered this question]

Out of 27 symptomatic respondents, 20 chose not to test due to less severity. However, 7 responded that they were restrained by the cost of the test. No person reported getting denied a test.

Summarised Result:

27 out of 63 respondents (35\%) reported having cold, dry cough, and or fever, from March 15 to June 15.

Out of 27 symptomatic participants, only 1 reported to have been tested for Covid- 19 .

$80 \%$ of symptomatic participants chose to not test, solely based on the decreased severity of symptoms.

7 respondents showed severe symptoms but restrained themselves from taking a test due to the high cost of testing for Covid-19.

$74 \%$ of symptomatic respondents did not take measures to isolate themselves, or practice self quarantine.

\section{Conclusion and key take-aways:}

There is a rampant need to educate people on the importance of self isolation, if they are showing symptoms of Covid-19. WHO recommends a 14 day self quarantine if there are symptoms that indicate chances of a positive Covid-19 case.

Testing should be made more accessible, and people should be encouraged to get tested after consulting a physician if they are showing common symptoms of Covid-19.

Cost of testing remains a deciding factor in a developing country, efforts should be directed at reduction in cost of testing. 
Daily testing data in India currently stands at 0.11 tests per 1000 people. This is one of the lowest in the world, and there exists a stark need to rapidly increase the number of tests.

\section{Bibliography:}

https://ourworldindata.org/coronavirus-testing

https://www.who.int/emergencies/diseases/novel-coronavirus-2019/advice-for-public

https://ourworldindata.org/coronavirus-testing 